\title{
Research and Practice of "Fault-Based" Experimental Teaching Mode
}

\author{
Xiulei Zhang \\ School of Automation Science and Electrical Engineering \\ Beihang University \\ Beijing, China, 100191
}

\author{
Changbo Fan* \\ School of Automation Science and Electrical Engineering \\ Beihang University \\ Beijing, China, 100191 \\ *The corresponding author
}

\author{
Xingming $\mathrm{Wu}$ \\ School of Automation Science and Electrical Engineering \\ Beihang University \\ Beijing, China, 100191
}

\begin{abstract}
In the light of the phenomena that students deliberately avoid the experiment fault, negatively cope with experimental problems and do poor in circuit debugging and troubleshooting, this paper put forward a new fault-based experimental teaching mode, and design a teaching framework concerning teaching materials and experiment report, experimental process, evaluation system, experiment assessment. Practice has proven that this teaching mode improves teachers teaching level and students' practical ability, deepens students' understanding and application of theoretical knowledge, improves their experimental mentality, and achieves good teaching results.
\end{abstract}

Keywords-experiment teaching; experimental failure; experimental teaching mode; experimental mentality

\section{INTRODUCTION}

Experimental and practical teaching is an indispensable part in engineering education, and the scientific and effective implementation of experimental teaching is the key to ensure the graduation of engineering professionals [1]. Electrical and electronic experiment, the common task in both electric and non-electric engineering professions in colleges and universities, is rich in content of varied scopes, and has strong practicality [2]. It mainly takes mature experimental projects as the carrier, to help students independently engage in experimental research, train their hands-on skills and improve their ability to analyze and solve problems, etc., which enables students to lay a solid foundation for the professional research in the future job. In order to tap the potential of experimental teaching, this paper studies and designs a novel fault-based experimental teaching mode, which can effectively change students' passive negative experimental mentality, and promote their understanding and application of theoretical knowledge, and cement the idea that experimental process is more important than the results.

Fund project: Beihang University Double Hundred Project Quality Program 2018 (Grade A), No: 4003095

\section{SitUATION AND PROBLEMS OF EXPERIMENTAL TEACHING}

The traditional imparting teaching is very commonly adopted in experiments, especially the basic experimental course. The basic teaching idea is to verify the theories [3]. This teaching mode follows standardized experimental contents, operating methods and steps, as well as fixed data processing methods and scoring rules. With it, students are prone to mechanically complete the experiment according to the established experimental steps and methods, and record and process experimental data. To put it simple, when preparing for class, students shall analyze circuit; master the experimental steps and methods in accordance with the teaching material. But they have no idea about what accident will happen and do not prepare for the potential faults in experiment. In this way, when fault occurs in the experiment, most of the students will have the psychologies that "how could it happen as the circuit is identical with the sample in the textbook, or the circuit is the same with those of other students", or "something wrong happens in my circuit board or the device", resulting in the manic behavior, helplessness, and even despair. Finally, they have no choice but to wait for teachers to help analyze and eliminate problems. It is known that in the various design training and competitions, there exist the phenomena that college students only know how to design, but have no idea of debugging [4]. These problems are more or less caused by the traditional teaching mode, which limits students' way of thinking, suppresses their personality and learning enthusiasm, and results in the mentality that they are doing experiment for experiment's sake [5]. Over time, students begin to feel negative and afraid of experimental courses, so they may pray for a smooth experiment, seek for the equipment or device contribute to successful experiment, put more than one experiment set standby in case of accidents. All these are the deeds to deliberately avoid experiment faults and problems, the negative response to the question, which are not conducive to the cultivation of the students' ability to analyze and solve problems. 


\section{RESEARCH AND DESIGN OF NEW EXPERIMENTAL TEACHING MODEL}

As the saying goes, failure is the mother of success. Frustration makes progress. To some extent, experimental failures can help students better understand the experiment and cultivate their ability to analyze and solve problems. From the perspective of education and teaching, active and effective utilization of experimental failures is an important way to improve the quality of experimental teaching [6].

In this paper, the fault-based experimental teaching mode is a kind of reverse teaching mode, is the practice of the theory knowledge application, and it attaches much more importance to the experimental process than to the results [7-8]. It requires students to actively deal with the problems in the experiment, avoid passively accepting experiment and negatively coping fault, and encourages students make faults in experiment and analyze and solve them. In this way, it can deepen students' understanding of theories and their applications, broaden students' mindset, and at the same time train their ability to methodically solve problems in calm.

The new experimental teaching mode integrates the fault occurrence, positioning and elimination into the whole experimental teaching framework, as shown in Fig. 1.

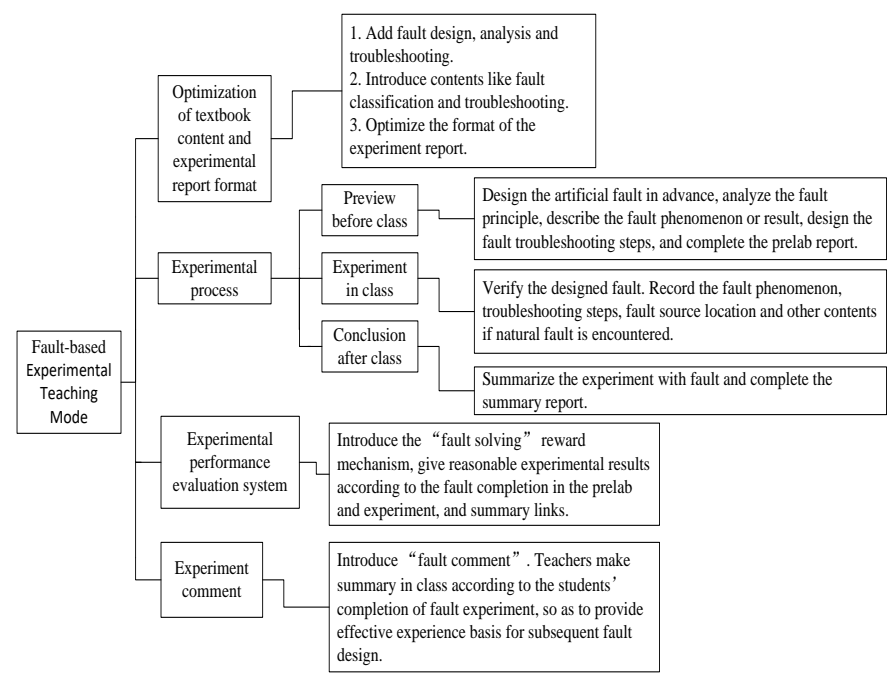

Fig. 1. The framework of experimental teaching mode based on fault

\section{A. Optimization of teaching material and experimental report format}

In order to cater to the new experimental teaching mode, the content of teaching materials and the format of experimental reports are optimized. Contents like the fault design essentials, fault analysis methods, and the general procedures of troubleshooting are added into the textbook to guide students to actively refer to materials, complete fault design on the premise of experimental principles and relevant theoretical knowledge, and conduct verification in the class, so as to better integrate theory and practice. Relevant contents of fault design are added to the experimental report, as shown in Fig. 2.

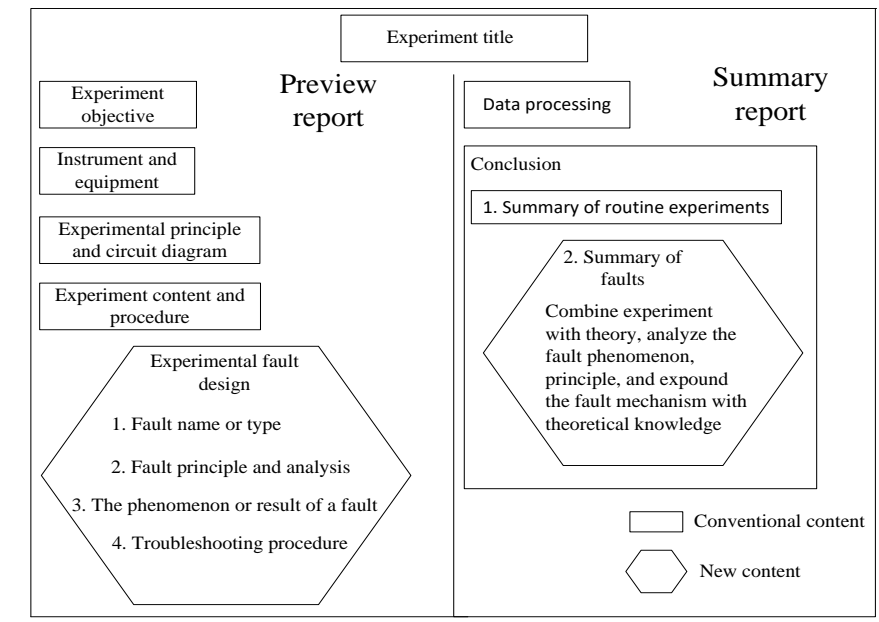

Fig. 2. Experiment report format

\section{B. Experiment procedure design}

\section{1) Preview before class}

The main common types of faults in electrical and electronic experiments are instrument failures and circuit failures. The instrument can be classified into two categories that is signal generation device and signal acquisition gadget. The former includes function signal generator and stabilized voltage supply, and the latter contains multimeter, oscilloscope and AC millivoltmeter.

As for instrument break-down, without damaging the equipment, one can make small troubles like cable break, error in instrument parameter setting, instrument in not common ground, which results in abnormal experimental results. As for the circuit fault, it is mainly incurred by changing the circuit component parameters, component categories, circuit structure, etc. Students should focus on circuit failure to better understand the principles of the circuit.

In the preview before class, students can analyze the experimental schematic diagram or circuit diagram according to the experimental content, design artificial fault in instrument fault or circuit fault with the theoretical knowledge, complete the fault principle analysis, fault phenomenon or result description, and troubleshooting step design, and then complete the experimental preview report. While carrying out pre-class simulation experiments, students are encouraged to use the simulation software Multisim which can visually demonstrate the working process of the circuit so as to facilitate students to conduct fault design. By simulating the designed fault circuit with simulation software, students can not only provide reference for the fault experiment in class, but also deepen their understanding of theoretical knowledge more effectively.

The number of experimental failures designed depends on the condition of experiment. Generally, students are required to produce 1 to 2 complex faults or 2 to 3 simple faults. In the complex faults, multiple changes may take place in intermediate test points caused by dramatic changes in experimental circuit principle parameters. In this respect, one needs to leverage a variety of instruments and combine with 
theoretical knowledge to locate the fault source. Whereas in simple faults, the parameters or structure of the experimental circuit and auxiliary circuit are changed slightly, resulting in fewer test points being influenced. Troubleshooting personnel can locate the fault source easily.

\section{2) Experiment in class}

In the classroom experiments, in addition to the routine experiment, students also need to complete the verification test of man-made faults, and sometimes they also need to complete the troubleshooting work of accident faults. For the verification test of man-made faults, students can complete the fault location through the phenomenon and the default troubleshooting steps, so as to achieve the fault-oriented learning purpose. For the inartificial fault encountered in the experiment, the students use their theoretical knowledge to analyze, troubleshoot and diagnose it. This can exercise the students' practical ability, improve the capability to analyze and solve problems [9].

Before the experiment, the teacher can carry out spot check on students' preview report, discuss the feasibility of the designed fault with students, and share the common problems with the class. When students meet the accident or finish setting troubles in the experiment, they should report to the teacher the fault type, phenomenon or the result. After screening out the source of trouble, students report the process and conclusion to the teacher who need to make the record throughout the experiment. Students themselves also need to complete the troubleshooting record for making the summary after class.

\section{3) The summary after class}

After class, students need to complete the summary report which should contain fault summary in addition to the conventional data processing and conclusion. The added fault summary mainly includes failure type, failure phenomenon, troubleshooting process and fault location analysis. Through learning the influence caused by the changes in circuit structure, parameter and test environment, students can deepen their understanding of the circuit principle from both theoretical and practical perspectives. At the same time, they will do better in problem discovering, analyzing and solving.

When reviewing the experiment report, teachers can also check the part of fault, including fault design and fault type selection, the inappropriate troubleshooting methods or procedures, and incomplete fault mechanism analysis, etc. The corresponding guidance or advice can be provided to help students understand and master relevant theoretical knowledge and experimental skills. Students should communicate with the teacher in time if they have any puzzles about the reviewed report.

\section{Design of the evaluation system of experimental results}

A Scientific experiment result evaluation system is the necessary standard to evaluate student experimental design, practice, report, and comprehensive ability [10-11]. Therefore, a comprehensive experimental achievement evaluation system with "fault in experiment" as the orientation and "fault addressing" as the emphasis need to be created for the implementation of new experimental teaching pattern guides based on the obstacles.

It the evaluation system, the experimental results of students in this system are from the usual experiment and the experimental operation test, which respectively account for $80 \%$ and $20 \%$ of the total scores. The usual experimental results are composed of three parts: the experimental preview report, the experiment process, and the summary report. The results of the experimental preview report are mainly evaluated according to the analysis of the experimental circuit principle, simulation, experimental step design and experimental fault design. The part of fault design accounts for $10 \%$ and the other $5 \%$. The results of the experimental process account for a large proportion. The students are mainly graded according to the experimental operation process in class, the proficiency of instrument and equipment operation, and the completion of the experimental content. The proportion of routine experiment is $20 \%$, and the proportion of fault experiment is $30 \%$ (inartificial fault accounts for $18 \%$, and man-made fault accounts for $12 \%$ ). The results of the experimental summary report are mainly graded by the experimental data processing, conclusion and theoretical analysis, with the summary of routine contents accounting for $5 \%$ and the summary of failure accounting for $10 \%$. The test score of experimental operation accounts for $20 \%$, mainly from students' understanding of the circuit principles, the correctness and proficiency in circuit connections and the use of instruments, as well as the examination of students' ability to find, analyze and cope with faults. The comprehensive experimental performance evaluation system is shown in Fig. 3.

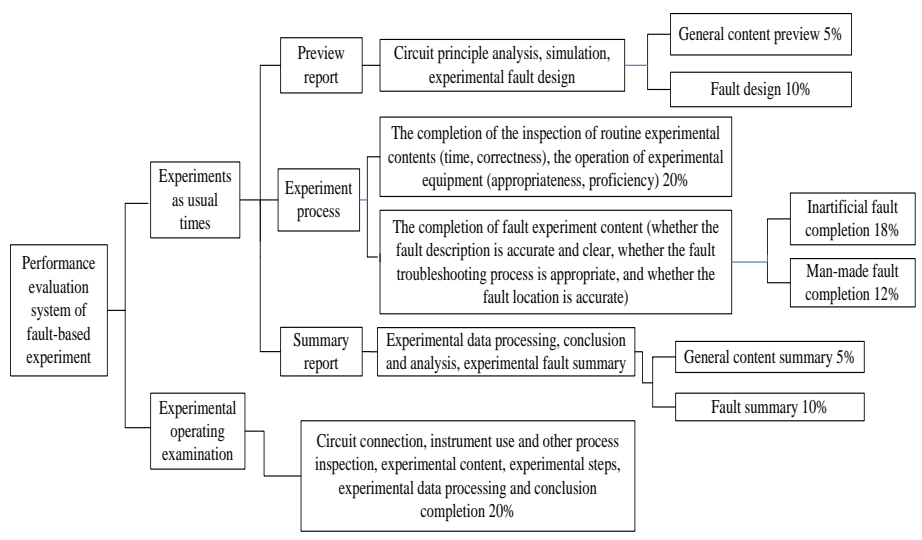

Fig. 3. The comprehensive experimental performance evaluation system

The comprehensive experiment achievement evaluation system, no longer takes speed and accuracy in the experiments as the only standard to assessment. It introduces reward mechanism on "fault design" and "troubleshooting", so as to inspire students to use the "fault" to apply and master the theoretical knowledge. In the process, students not only raise the ability to analyze and deal with obstacles, but also cultivate good mentality for experiment as they realize that process is more important than the results, and the setback makes progress, avoiding impatience and disorder in the experimental operation [12]. 


\section{Experimental review system design}

With the "experiment comment" mechanism introduced, teachers can summarize and comment on the last experiment in each experimental class, pointing out improper and wrong experiment results, analysis and operation method for students. At the same time, the experiment cases with accurate data processing, thorough analysis and effective methods are delivered to students. In the mechanism, "fault review" is of particular importance. Teachers conclude the fault design and experiment, and share with students several typical fault cases, which not only deepen students understanding and application of relevant theoretical knowledge, but also expand the students thinking vision.

\section{PRACTICE EFFECT}

In Beihang University, the electric technology practice foundation is a basic experimental course for the second and third grade undergraduates majoring in science and engineering. It is a practical teaching course including contents of "circuit", "analog electronic circuit" and "digital electronic circuit". Having been applied to this course for several semesters, the fault-based experimental teaching mode achieved good teaching effects as follows, judged by the teaching situation of several teachers in the course group and the feedback information of students.

(1) Students can access to a wide range of knowledge and witness diversified experimental phenomena and faults. The teaching mode also puts higher requirements on teachers' theoretical level and practical experience to force them to improve their teaching [13]. The theoretical knowledge of "point, line and surface" is organically linked together to build a strong and solid knowledge network, laying a solid foundation for future engineering practice

(2) The practice of the new teaching mode changes students' wrong view of experimental class. They will come to know that experiment is not simply carried to verify some theory, but actually the opportunity for students to leverage multiple knowledge points to analyze and interpret what happened in the experiments. With the changes in one or a series of circuit structure, students can use theory to guide the practice and in turn, utilize practice to test the theory. In the process, they not improve the practical ability, but also broaden their visions. By organically linking

(3) The fault-based teaching mode paves the way for students to compete in various matches or the activities off campus. For example, the chances of acceptance can be dramatically increased if students tell the interviewer what they have acquired in the experimental classes, like the experience in fault analyzing and troubleshooting. In terms of practice results, a questionnaire survey has been conducted on students who had participated in the National Undergraduate Electronics Design Contest, Feng Ru Cup Science and Technology Competition, off-campus engineering practice or some projects. These students, in generally, consider that the excellent results they obtained are inseparable from teachers' guidance and their accumulated practical experience in experimental classes. The new teaching mode is well recognized by both teachers and students.

\section{CONCLUSION}

The fault-based experimental teaching mode can not only help students cement the theoretical knowledge they have acquired, but also provide students the opportunities to experience the problem solving similar to the actual engineering and practical jobs. It takes the experimental fault as the driving point or starting point, and cultivates students' ability to analyze, find and troubleshoot faults with theoretical knowledge, testing tools and reasonable and effective testing methods. Taking obstacles in the experiment as the starting point, we can instruct students to make use of theoretical knowledge, test tools and proper test methods, which can cultivate their ability to analyze, locate and eliminate faults. This teaching method broadens students' horizon and thinking, and strengthens their confidence and competitiveness, laying solid foundation for their future jobs or the postgraduate studies.

\section{REFERENCES}

[1] Tian Lixin, Gao Zhiqi. Research on Reform of Electrics and Electronics Experiment Teaching Oriented to Engineering Education[J]. Journal of Electrical and Electronic Engineering Education, 2010, 30(Supplement 2):159-161.

[2] Li Wen. Research on Electrical and Electronic Experiment Teaching under Engineering Background[J]. Experimental Technology and Management, 2012, 29(4):254-257.

[3] Zheng Hong, Yu Shouqian. Investigation and Practice of Experiment Teaching Method Based on "Active Obstacle Setting”[J].Journal of Beijing University of Aeronautics and Astronautics, 2009, 22(Supplement):46-50.

[4] Li Heng, Shao Jianlong, Zhang Guoyin et al. Exploration and Practice of Electrical and Electronic Trouble Clearing Experimental Teaching Model[J]. Computer Knowledge and Technology, 2012, 8(7):1715-1717.

[5] Li Ming. Research and Practice of Designing Experiment Teaching[J].Experimental Technology and Management, 2012, 29(4): 245-247.

[6] Hao Dapeng, Chen Hua, Xun Zhipeng. Experimental Fault Plays An Active Role in Physics Experiment Teaching[J]. Education Forum, 2013 (52):278-279.

[7] Lv Nianling, Yuan Yancheng, Huang Xiaomei et al. The Importance of Experiment Teaching Be in Process[J].Experimental Technology and Management, 2017, 34(6): 154-156.

[8] Li Jing, Xia Youwei. The Importance of Experiment Teaching Be in Process[J]. Research and Exploration in Laboratory, 2009, 28(9):1-4.

[9] Zhou Gengkuan. The practice of troubleshooting experimental teaching method[J]. Research and Exploration in Laboratory, 1994(4):5-6.

[10] Ning Tao. Design and practice of research-based practical teaching[J]. Experimental Technology and Management, 2014, 9(31):284-286.

[11] She Yuanfu, Wang Qingren. Constructing Evaluation of ResearchOriented Teaching in Higher Institutions[J]. Research in Higher Education of Engineering, 2011(6): 111-115.

[12] Zhang Xiumin, Zhu Ying, Pu Xiaowen et al. Discussion on Experimental Teaching Model of Digital Electronic Technique[J]. Experimental Technology and Management, 2014, 9(31):298-300.

[13] Wang Xingang, He Li, Zhang Dongzhi at al. Reform and Practice of Research-Oriented Experiment Teaching Mode in the Course of Electrical and Electronics[J]. Research and Exploration in Laboratory, 2017, 36(4):188-191. 